\title{
Quel enseignement la médecine peut-elle tirer de la biologie des systèmes?
}

Président de conférence dans le cadre de l'assemblée annuelle de

Directeur Klinik und Poliklinik

für Innere Medizin

UniversitätsSpital Zürich

\section{Edouard Battegay} la SSMI en 2013,
Correspondance:

Prof. Dr Edouard Battegay

Klinik und Poliklinik

für Innere Medizin

UniversitätsSpital Zürich

Rämistrasse 100

CH-8091 Zurich
Au cours des dernières décennies, la biologie a connu un développement fulgurant. De nouvelles molécules ont été découvertes dans les cellules et leur fonction a été décryptée. Grâce à une spécialisation, les connaissances de nombreux biologistes n'ont cessé de s'enrichir. Ainsi, le savoir s'est accru. Pourtant, souvent, de nombreux éléments individuels ne fournissaient pas de tableau d'ensemble satisfaisant. De nombreux processus biologiques peuvent uniquement être compris en connaissant non seulement la fonction d'une molécule donnée, mais également l'interaction entre différents processus cellulaires.

Dans la biologie, un domaine de recherche précisément consacré à cette synergie des différents facteurs individuels s'est dès lors développé au cours de ces dernières années: la biologie des systèmes. «La biologie des systèmes est nécessaire pour comprendre certains processus, mais elle ne remplace pas la recherche spécialisée», affirme le Prof. Rudolf Aebersold, Professeur de biologie des systèmes à l'ETH de Zurich. «Il faut considérer les processus biologiques à une plus vaste échelle pour avancer.»

Le Prof. Rudolf Aebersold présentera les développements dans la biologie à l'occasion de la President's Lecture de l'assemblée annuelle de la SSMI. Il montrera comment il étudie dans son travail l'interconnexion des différentes molécules. Un biologiste des systèmes est un "généraliste» de la biologie. Contrairement aux spécialistes, il connaît un plus grand nombre de processus biologiques, mais moins en détails. Le biologiste des systèmes doit également être familiarisé avec les méthodes de travail de domaines de recherche voisins, comme par ex. l'informatique, même s'il ne maitrise pas le domaine aussi bien qu'un spécialiste en informatique. Le biologiste des systèmes a donc pour mission de comprendre les connexions entre les variables individuelles.

\footnotetext{
Assemblée annuelle de la SSMI en 2013 -

Thèmes: la multimorbidité et la nouvelle structure du programme

Le problème des maladies chroniques et de la multimorbidité va croissant et il sera au cœur de l'assemblée annuelle de la SSMI du 29 au 31 mai 2013 à Bâle. Le sujet y sera abordé sous un angle médical et les conséquences de la multimorbidité au niveau de la planification des soins seront évaluées. La structure du programme sera modifiée en 2013. L'objectif est de rendre le programme nettement plus compréhensible et de répondre aux besoins variés des participants. Des informations supplémentaires sont disponibles sur www.congrex. $\mathrm{ch} / \mathrm{fr} /$ sgim2013 ou www.sgim.ch/fr/manifestations-de-formation-continue/ ssmi-congres-annuel/
}

Dès lors, un véritable changement de paradigme s'est produit dans différents domaines au cours de ces dernières années. Dans la biologie, il est de plus en plus admis qu'il ne suffit pas de connaître tous les facteurs individuels pour pouvoir prédire la fonction de l'ensemble. Ce changement de paradigme peut être démontré en prenant l'exemple de l'évolution dans le domaine de la recherche génomique. Dans les années 1990, il régnait une euphorie car on pensait pouvoir prédire la survenue de nombreuses maladies grâce au séquençage du génome humain. Après le séquençage du génome humain, le génome des hominidés a également été décrypté en partant du principe que l'on pourrait ensuite démontrer ce qui distingue l'homme du singe. Ce paradigme n'a durant longtemps pas été remis en question. Toutefois, il est aujourd'hui admis que les rapports sont plus complexes qu'il n'y paraissait: une mission pour la biologie des systèmes.

Des recherches génomiques intensives ont également été conduites dans le domaine de l'oncologie. Dans ce domaine non plus, la simple relation «une mutation induit une carcinogenèse» n'a pas pu être établie. Les génomes de différentes cellules cancéreuses de centaines de patients ont été comparés. Il n'y avait pas une seule mutation qui était toujours présente pour une forme de cancer déterminée. Par contre, il a été possible d'identifier certains modèles de répartition d'altérations génétiques, qui étaient corrélées avec certaines formes de cancers. Actuellement, la recherche en biologie des systèmes tente par ex. de déterminer comment la variabilité génomique se répercute sur la cellule. La survenue d'un cancer est l'une des répercussions potentielles sur la cellule. Il reste à déterminer comment des facteurs environnementaux ou d'autres facteurs influencent la cellule et via quels réseaux la réaction cellulaire se produit.

La promotion de la biologie des systèmes a également déclenché des craintes chez des biologistes relativement spécialisés. "Inutilement», pense le Prof. Aebersold. «La recherche se poursuit à différents niveaux, dans la spécialisation comme dans la biologie des systèmes.»

Pour les participants à l'assemblée annuelle de la SSMI, il sera sans doute intéressant d'apprendre comment la biologie spécialisée et la biologie générale se développent et quelles connaissances en découlent. Des impulsions essentielles pour la médecine peuvent s'ensuivre. 